\title{
Density profile evolution and nonequilibrium effects in partial and full spreading measurements of surface diffusion
}

\author{
Nikunen, P.; Vattulainen, Ilpo Tapio; Ala-Nissila, T.
}

Published in:

Journal of Chemical Physics

Link to article, DOI:

$10.1063 / 1.1355765$

Publication date:

2001

Document Version

Publisher's PDF, also known as Version of record

Link back to DTU Orbit

Citation (APA):

Nikunen, P., Vattulainen, I. T., \& Ala-Nissila, T. (2001). Density profile evolution and nonequilibrium effects in partial and full spreading measurements of surface diffusion. Journal of Chemical Physics, 114(14), 6335-6342. https://doi.org/10.1063/1.1355765

\section{General rights}

Copyright and moral rights for the publications made accessible in the public portal are retained by the authors and/or other copyright owners and it is a condition of accessing publications that users recognise and abide by the legal requirements associated with these rights.

- Users may download and print one copy of any publication from the public portal for the purpose of private study or research.

- You may not further distribute the material or use it for any profit-making activity or commercial gain

- You may freely distribute the URL identifying the publication in the public portal 


\title{
Density profile evolution and nonequilibrium effects in partial and full spreading measurements of surface diffusion
}

\author{
P. Nikunen \\ Helsinki Institute of Physics and Laboratory of Physics, Helsinki University of Technology, \\ P.O. Box 1100, FIN-02015 HUT, Espoo, Finland \\ I. Vattulainen ${ }^{\mathrm{a})}$ \\ Department of Chemistry, Technical University of Denmark, Building 207, DK-2800 Lyngby, Denmark
}

T. Ala-Nissila

Helsinki Institute of Physics and Laboratory of Physics, Helsinki University of Technology, P.O. Box 1100, FIN-02015 HUT, Espoo, Finland, and Department of Physics, Brown University, Providence, Rhode Island 02912-1843

(Received 18 September 2000; accepted 24 January 2001)

\begin{abstract}
We study the nature of nonequilibrium effects in the collective diffusion coefficient $D_{C}(\theta)$ vs the coverage $\theta$ as extracted from Boltzmann-Matano analysis of spreading coverage profiles. We focus on the temporal behavior of the profiles and study how the corresponding nonequilibrium effects in $D_{C}(\theta)$ depend on the initial density gradient and the initial state from which the spreading starts. To this end, we carry out extensive Monte Carlo simulations for a lattice-gas model of the O/W(110) system. Studies of submonolayer spreading from an initially ordered $p(2 \times 1)$ phase at $\theta=\frac{1}{2}$ reveal that the spreading and diffusion rates in directions parallel and perpendicular to rows of oxygen atoms are significantly different within the ordered phase. Aside from this effect, we find that the degree of ordering in the initial phase has a relatively small impact on the overall behavior of $D_{C}(\theta)$. Also, although we find that nonequilibrium effects are clearly present in submonolayer spreading profiles, $D_{C}(\theta)$ determined from such data approaches its asymptotic equilibrium behavior much more rapidly than in the case of full spreading. Nevertheless, in both cases there are noticeable deviations from equilibrium results that persist even at very long times and are strongest in ordered phases and in the vicinity of phase boundaries. These conclusions are confirmed by complementary studies of the temporal behavior of the order parameter $\phi(\theta)$. Finally, we use $D_{C}(\theta)$ and $\phi(\theta)$ to determine the locations of phase boundaries and find such data to be clearly time dependent during full spreading. We conclude that nonequilibrium effects seem to be an inherent feature in profile evolution studies of surface diffusion in all cases where ordering plays a prominent role. This warrants particular care to be taken with profile spreading experiments. (C) 2001 American Institute of Physics. [DOI: 10.1063/1.1355765]
\end{abstract}

\section{INTRODUCTION}

The transport of adatoms and molecules on surfaces plays a major role in various physical processes such as the formation of nanostructures via self-assembly or cluster deposition, chemical reactions, and spreading of thin films. ${ }^{1-3}$ The fundamental as well as technological interest for understanding the basic principles that govern surface diffusion is therefore evident. This has motivated a lot of experimental and theoretical activities ${ }^{1-8}$ which have yielded intriguing insight in the diffusion characteristics in many surface adsorbate systems. This includes studies of the microscopic mechanisms by which adparticles and clusters move along the surface, and how the diffusion rates are influenced by interaction effects between adparticles. However, one of the long-standing problems in experimental studies of surface diffusion regards a variety of experimental techniques, whose results even for the same surface systems may

${ }^{\text {a)} E l e c t r o n i c ~ m a i l: ~ i l p o . v a t t u l a i n e n @ c s c . f i ~}$ strongly depend on the technique used. ${ }^{2,6}$ This is relatively easy to understand in cases where one compares methods which probe the diffusion process strictly in equilibrium with other techniques that operate under far-from-equilibrium conditions. Various experimental and theoretical studies have shown ${ }^{5,6,9-14}$ that such nonequilibrium measurements may yield results that are distinctly different from the equilibrium ones.

An intriguing situation arises when there are only slight deviations from equilibrium, in the sense that one might expect the assumption of local equilibrium to be satisfied, and the linear response theory leading to the diffusion equation to be valid. This is typically the case in profile evolution measurements of surface diffusion, ${ }^{4,15-21}$ where the equilibrium theory is often used to analyze the measurements. In situations where there are no ordered phases present, recent careful studies have shown ${ }^{21,22}$ that very good agreement with the equilibrium results can be obtained when spreading occurs in the submonolayer regime without ordering effects. 
However, in the case of strong interactions leading to ordered phases, experiments clearly indicate ${ }^{18,20}$ that the diffusion behavior can be affected by nonequilibrium effects, leading to diffusion coefficients that depend on the time scale during which the spreading data are collected.

A case in point is the well-established BoltzmannMatano (BM) method, ${ }^{6,23,24}$ which is commonly used ${ }^{4,14-22,25}$ to extract the collective diffusion coefficient $D_{C}(\theta)$ as a function of coverage $\theta$ from scaled coverage (density) profiles. We have recently shown ${ }^{26}$ through Monte Carlo simulations of a lattice-gas model of $\mathrm{O} / \mathrm{W}(110)$ that nonequilibrium effects can play a noticeable role under conditions where there are several ordered phases present at different coverages. In Ref. 26, we focused on the temporal behavior of $D_{C}(\theta)$ as the system approaches equilibrium starting from a step function density profile at a full initial coverage of $\theta=1$. We demonstrated that the effective $D_{C}(\theta)$ and the corresponding diffusion barriers obtained in this fashion depend strongly on the time regime chosen for analysis of the density profiles, and may deviate significantly from results obtained from equilibrium simulations especially within ordered phases and close to phase transition boundaries. This is essentially due to the interplay between spreading and phaseordering kinetics, and is manifested in the particle number fluctuations that differ significantly from their equilibrium value. ${ }^{26}$

In the present work, we consider the influence of nonequilibrium effects in partial spreading experiments where the initial coverage is less than unity. In particular, we consider the temporal behavior of the effective collective diffusion coefficient $D_{C}(\theta)$ within and below the ideal $p(2 \times 1)$ phase in the lattice-gas model of $\mathrm{O} / \mathrm{W}(110)$. In this case, we apply the Boltzmann-Matano analysis to profiles extracted from initially steplike coverage profiles at a fixed coverage of $\theta=\frac{1}{2}$, where the degree of ordering within the initial phase is varied to consider its influence on $D_{C}(\theta)$. The results are then compared with the full spreading case where the initial coverage $\theta=1 .{ }^{26}$ We find that while nonequilibrium effects are still clearly present, $D_{C}(\theta)$ as determined from the partial spreading case approaches its asymptotic equilibrium behavior much more rapidly than in the case of full spreading. We also find that the spreading and diffusion rates in directions parallel and perpendicular to rows of ogygen atoms are significantly different within the ordered $p(2 \times 1)$ phase. Aside from this effect, the degree of ordering in the initial phase at $\theta=\frac{1}{2}$ has a relatively small impact on the behavior of $D_{C}(\theta)$. These conclusions are supported by the temporal behavior of the order parameter $\phi(\theta)$, which is used to quantify deviations from equilibrium during spreading. Finally, we use $D_{C}(\theta)$ and $\phi(\theta)$ to study the locations of phase boundaries. We find that the phase boundaries, as determined from these data, show clear time dependence in the case of full spreading. At early times the effective locations of phase boundaries deviate significantly from their equilibrium counterparts, while at long times the deviation becomes negligible. In submonolayer studies these effects are present but considerably weaker. Based on our Boltzmann-Matano studies, we can conclude that nonequilibrium effects seem to be an inherent feature in all spreading studies of surface diffu-

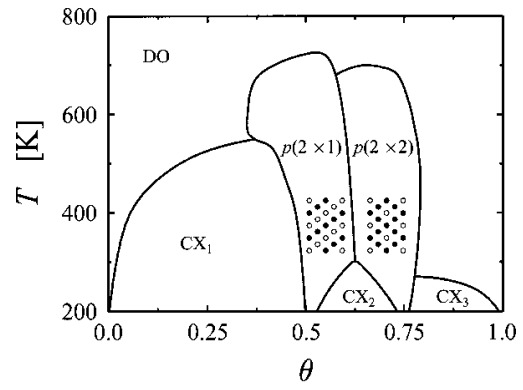

FIG. 1. Phase diagram of the lattice-gas model of the O/W(110) system. DO denotes the disordered phase, $p(2 \times 1)$ and $p(2 \times 2)$ denote the ordered phases at intermediate coverages, and $\operatorname{CX}_{i}(i=1,2,3)$ are the various lowtemperature coexistence phases. Configuration snapshots of ideal ordered phases are also shown, the occupied and vacant adsorption sites being denoted by filled and open circles, respectively.

sion in ordered phases. Predicting the correct equilibrium behavior of $D_{C}(\theta)$ therefore requires one to analyze the data at very long times. This warrants particular care to be taken when profile spreading experiments are being carried out.

\section{MODEL AND METHOD}

The lattice-gas Hamiltonian employed in this work is a model of the $\mathrm{O} / \mathrm{W}(110)$ adsorption system. The interaction parameters have been $\operatorname{chosen}^{27,28}$ such that the resulting phase diagram shown in Fig. 1 is in close agreement with the experimental observations. ${ }^{29}$ In the present study we concentrate on the coverage dependence of $D_{C}$ at a temperature of $T=590 \mathrm{~K}$ to allow direct comparison to the equilibrium results for $D_{C}(\theta),{ }^{27,28}$ and to results obtained from Boltzmann-Matano analysis of full spreading. ${ }^{26}$ At $T$ $=590 \mathrm{~K}$, starting from the coverage $\theta=\frac{1}{2}$ corresponding to the ideal $p(2 \times 1)$ phase, the system undergoes a continuous phase transition around $\theta \approx 0.37$ to a disordered (DO) phase at low coverages. ${ }^{30} \mathrm{We}$ wish to emphasize that the model has been extensively studied, and a complete set of equilibrium data for diffusion coefficients and other relevant quantities at various temperatures can be found elsewhere. ${ }^{27,28}$

As is well known, a proper choice of the transition probabilities within the Monte Carlo method is important to guarantee realistic description of surface diffusion. ${ }^{31-33}$ In particular, the thermally activated nature of surface diffusion processes must be described in a physically sensible fashion. In view of recent studies, ${ }^{31,33}$ where the behavior of surface diffusion coefficients was found to be sensitive to the choice of dynamics in Monte Carlo, this issue should clearly be given more attention.

In our Monte Carlo simulations, we employ the transition dynamics algorithm ${ }^{26,27}$ where single-particle jumps are described by transition probabilities $w_{i, f}$ from an initial state $i$ with energy $E_{i}$ to a final state $f$ with energy $E_{f}$, and proceed by two successive steps via an intermediate "saddle point" or transition state $I$ with energy $E_{I}=\Delta+\left(E_{i}+E_{f}\right) / 2$ such that $w_{i, f}=w_{i, I} w_{I, f}$. The rates have a Metropolis form $w_{i, j}=\min \left[1, \exp \left(-\left(E_{j}-E_{i}\right) / k_{B} T\right)\right]$ and the quantity $\Delta>0$ characterizes the effect of the (bare) saddle point of the adiabatic substrate potential. Thus for $\Delta>0$, the rates can be of activated form also for jumps with $E_{i} \geqslant E_{f}$. We note that a 
few other schemes, in which the idea of thermally activated diffusion processes has also been accounted for, have been suggested in Refs. 8 and 34. The use of TDA is furthermore supported by recent molecular dynamics studies, ${ }^{31,32}$ where it was found that TDA is qualitatively consistent with the dynamics seen in a true microscopic model of a system consisting of interacting particles. The time is defined in terms of one Monte Carlo step (MCS), during which every particle attempts to jump once on the average. Further details and additional references can be found in Ref. 27.

We consider the spreading of a coverage profile $\theta(x, t)$ in a semi-infinite system, which ranges from $-\infty$ to $+\infty$ in the $x$ direction, and whose width $L_{y}$ is typically 30 to 1000 lattice units in the $y$ direction. The exact system size used is mentioned below when necessary. Periodic boundary conditions are employed in the $y$ direction. The coverage profile is initially an ideal step function at $x=0\left[\theta(x, 0)=\frac{1}{2}\right.$ for $x<0$ and $\theta(x, 0)=0$ for $x>0$ ], which evolves in time $t$ in the $+x$ direction. Note that the coordinate $x$ also acts as the dividing line $x=0$, which accounts for particle number conservation via $\int_{0}^{1 / 2} x\left(\theta^{\prime}\right) d \theta^{\prime}=0$.

To determine the collective diffusion coefficient $D_{C}(\theta)$, we use the Boltzmann-Matano method ${ }^{6,23,24}$ as in Ref. 26, where $D_{C}(\theta)$ can be determined from scaled density profiles as

$$
D_{C}(\theta)=-\left.\frac{1}{2 t}\left(\frac{d x}{d \theta^{\prime}}\right)\right|_{\theta} \int_{0}^{\theta} x\left(\theta^{\prime}\right) d \theta^{\prime} .
$$

Note that the Boltzmann-Matano method is based on the assumption that, in the long-time limit, the coverage profiles $\theta(x, t)$ collapse to a single scaling function when expressed as $\theta(x / \sqrt{t})$. If this condition is truly satisfied, $D_{C}(\theta)$ obtained from Eq. (1) corresponds to the actual diffusion coefficient in equilibrium. Otherwise, the effective $D_{C}(\theta)$ in Eq. (1) is a nonequilibrium quantity and depends on a time regime chosen for an analysis.

In equilibrium at low temperatures, the initial coverage $\theta(x<0,0)=\frac{1}{2}$ is characterized by the ordered $p(2 \times 1)$ phase that has two degenerate ground states. It is spatially anisotropic and thus there are two possible directions for spreading from a given ideally ordered phase. We adopt a notation here according to which the directions perpendicular and parallel to the atomic rows of oxygen atoms are denoted by $x_{\perp}$ and $x_{\|}$, respectively, as shown in Figs. 2(a) and 2(b). In Figs. 2(a)-2(d) we show the four different initial states from which the spreading process is let to evolve in time:

(a) Ordered $p(2 \times 1)$ phase. The phase is first equilibrated with periodic boundary conditions and then let to spread in the $x_{\perp}$ direction.

(b) Ordered $p(1 \times 2)$ phase. The phase is first equilibrated with periodic boundary conditions, after which spreading proceeds in the direction of $x_{\|}$.

(c) A mixture of equilibrated and ordered $p(2 \times 1)$ and $p(1 \times 2)$ domains of equal size. At $t=0$, the two phases are separated by a sharp interface at $y=L_{y} / 2$.

(d) Totally disordered initial state at $\theta=\frac{1}{2}$. This case corresponds to a situation in many experiments, where the initial steplike profile is made by depositing particles to

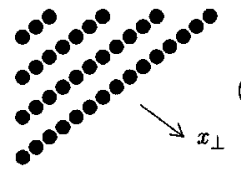

(a)

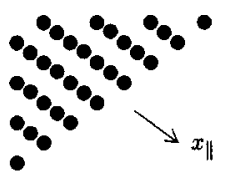

(b)

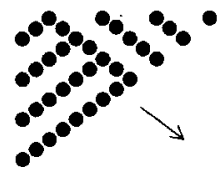

(c)

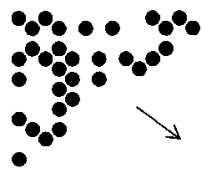

(d)

FIG. 2. A schematic figure on the initial coverage profiles we have used in submonolayer spreading studies: (a) fully ordered $p(2 \times 1)$ phase, (b) fully ordered $p(1 \times 2)$ phase, (c) a mixture of the $p(2 \times 1)$ and $p(1 \times 2)$ domains of equal size, and (d) a fully disordered system. All initial coverage profiles are for $\theta=\frac{1}{2}$. In the case of (a) and (b), we have also illustrated the directions perpendicular $\left(x_{\perp}\right)$ and parallel $\left(x_{\|}\right)$to the rows of oxygen atoms.

a surface at a low temperature. The spreading process is then initiated by sudden heating to a higher $T$.

The fact that there are two possible directions for spreading also means that, within the ordered phase, the spreading rate and hence $D_{C}(\theta)$ will be somewhat different in cases (a) and (b). For forthcoming comparisons with submonolayer diffusion data, we therefore define the corresponding equilibrium quantities

$$
\begin{aligned}
& D_{C, \perp}(\theta)=D_{C}(\theta) \Gamma_{\perp}(\theta) / \Gamma(\theta), \\
& D_{C, \|}(\theta)=D_{C}(\theta) \Gamma_{\|}(\theta) / \Gamma(\theta) .
\end{aligned}
$$

This description is based on the fact that, within the latticegas model, $D_{C}$ is proportional to the average transition rate $\Gamma{ }^{27,28}$ Thus $D_{C}(\theta)$ in Eq. (2) is the collective diffusion coefficient calculated in equilibrium, and $\Gamma$ is the average transition rate of all possible single-particle jump attempts. $\Gamma_{\perp}$ and $\Gamma_{\|}$correspond to average transition rates of jump attempts in directions perpendicular and parallel to the rows of oxygen atoms, respectively. ${ }^{35}$

\section{RESULTS}

\section{A. Collective diffusion}

In Figs. 3(a) and 4(a) we show the temporal behavior of coverage profiles $\theta(x, t)$ at three different time regimes during the profile evolution process. These results serve to demonstrate how density gradients drive diffusion towards the $+x$ direction. Then in Figs. 3(b) and 4(b) we show the scaled coverage profiles $\theta(x / \sqrt{t})$ at three different time regimes during the profile evolution process, taken at the same instances of time as in Ref. 26 (10000 to $12000 \mathrm{MCS}$, 50000 to $60000 \mathrm{MCS}$, and 250000 to $300000 \mathrm{MCS}$ ). Slight deviations between these different curves are apparent in the insets of the figures. Figures 3(c) and 4(c) show the effective collective diffusion coefficients as extracted from these data, together with the corresponding equilibrium results. As can be seen from the results, even at the earliest times around $10000 \mathrm{MCS}$ the effective $D_{C}(\theta)$ is relatively close to the 


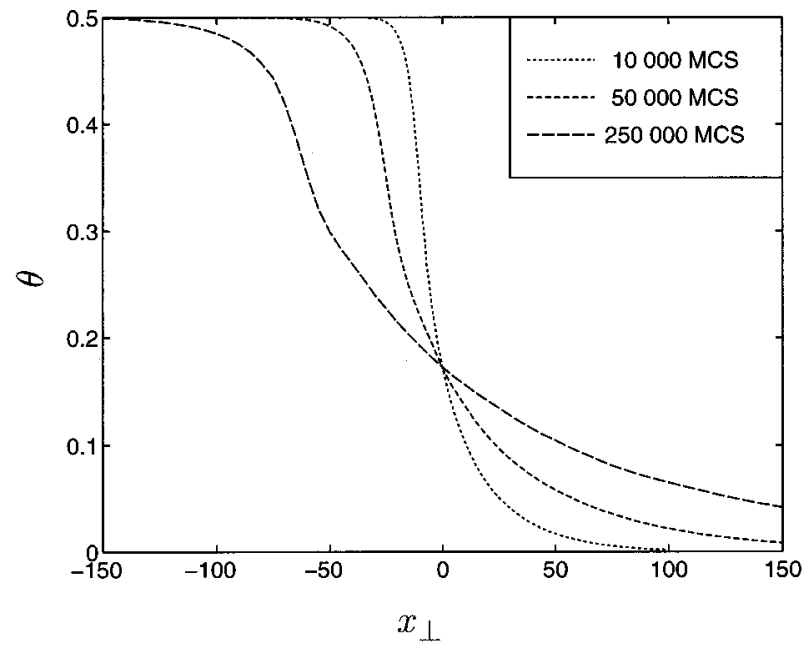

(a)

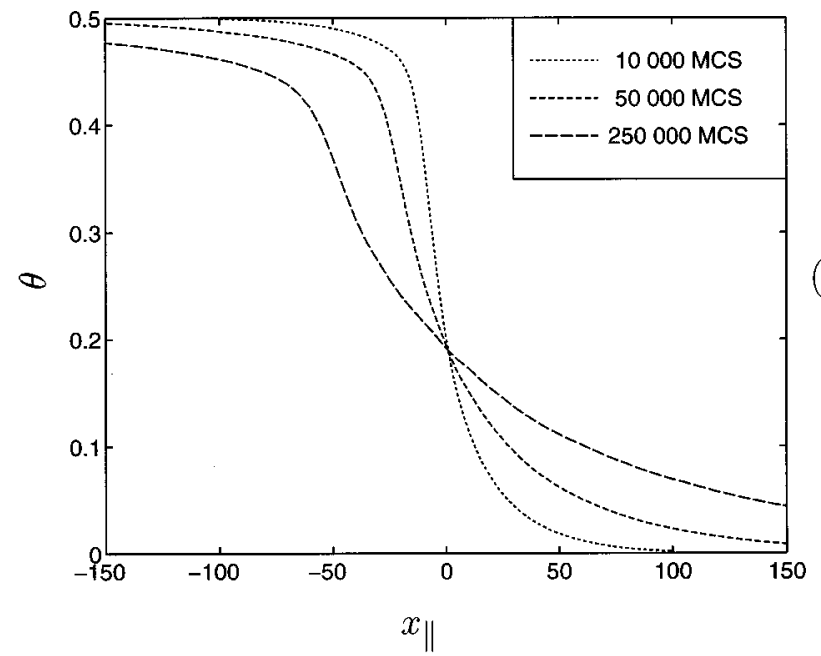

(a)

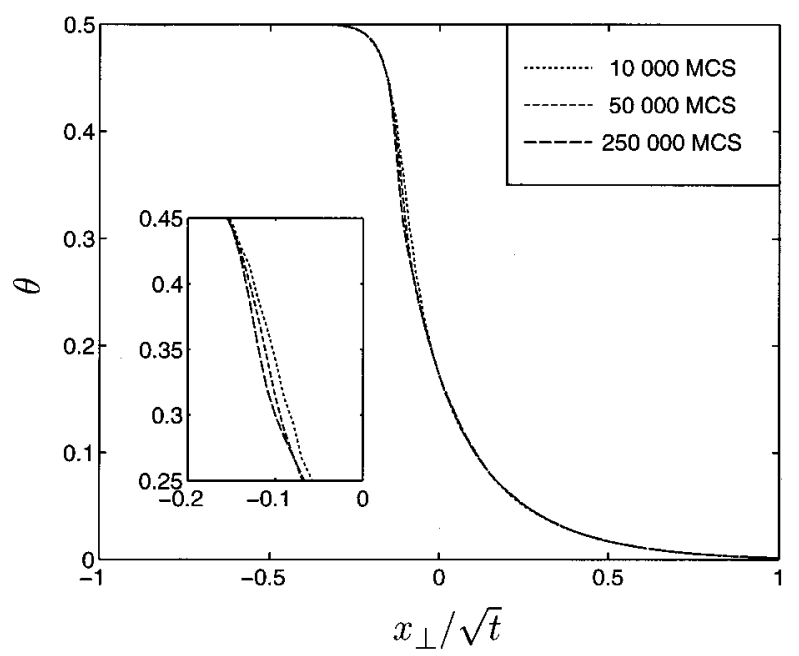

(b)
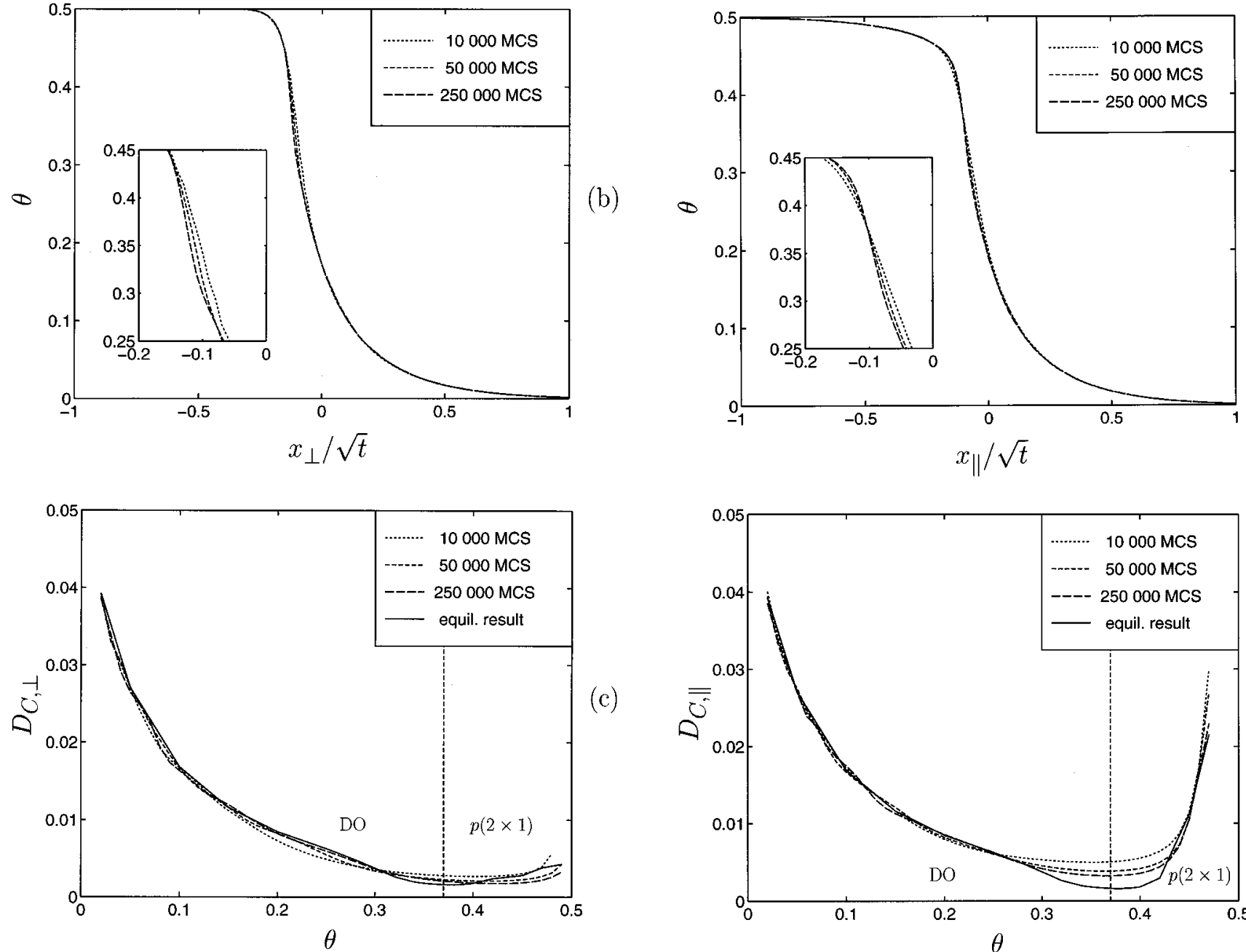

(b)

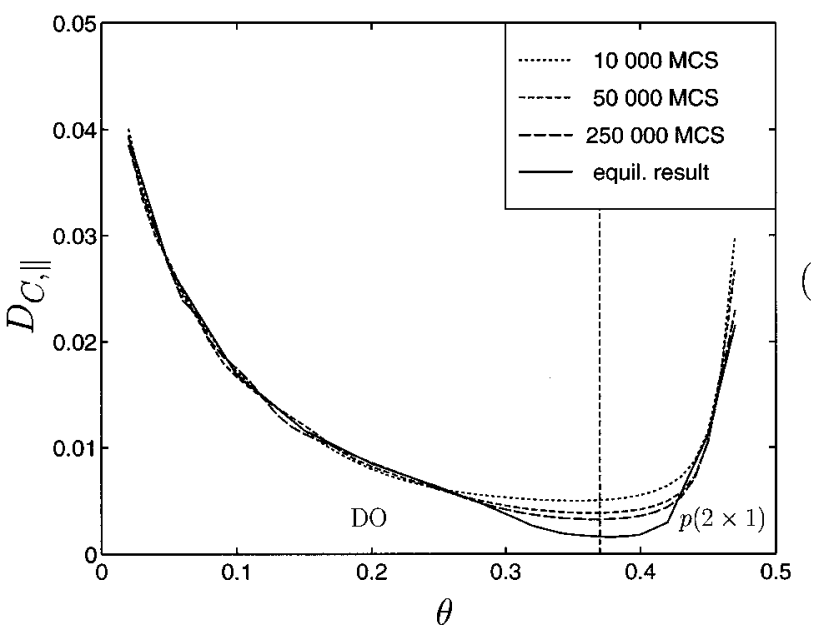

FIG. 3. (a) Coverage profiles $\theta\left(x_{\perp}, t\right)$ at $T=590 \mathrm{~K}$ at three different times during the partial profile evolution process starting from the ideally ordered $p(2 \times 1)$ phase. (b) Scaled coverage profiles $\theta\left(x_{\perp} / \sqrt{t}\right)$ at $T=590 \mathrm{~K}$ at three different times during the partial profile evolution process starting from the ideally ordered $p(2 \times 1)$ phase. In the data, several profiles from the time regimes 10000 to $12000 \mathrm{MCS}, 50000$ to $60000 \mathrm{MCS}$, and 250000 to 300000 MCS have been collapsed to obtain the scaled curves. The inset shows details of the profiles close to the phase boundary at $\theta \approx 0.37$. (c) The effective diffusion coefficients $D_{C, \perp}(\theta)$ as extracted from the profiles in (a). The solid line shows the asymptotic equilibrium result.

FIG. 4. (a) Coverage profiles $\theta\left(x_{\|}, t\right)$ at $T=590 \mathrm{~K}$ at three different times during the partial profile evolution process starting from the ideally ordered $p(1 \times 2)$ phase. (b) Scaled coverage profiles $\theta\left(x_{\|} / \sqrt{t}\right)$ at $T=590 \mathrm{~K}$ at three different times during the partial profile evolution process starting from the ideally ordered $p(1 \times 2)$ phase. In the data, several profiles from the time regimes 10000 to $12000 \mathrm{MCS}, 50000$ to $60000 \mathrm{MCS}$, and 250000 to 300000 MCS have been collapsed to obtain the scaled curves. The inset shows details of the profiles close to the phase boundary at $\theta \approx 0.37$. (c) The effective diffusion coefficients $D_{C, \|}(\theta)$ as extracted from the profiles in (a). The solid line shows the asymptotic equilibrium result. 


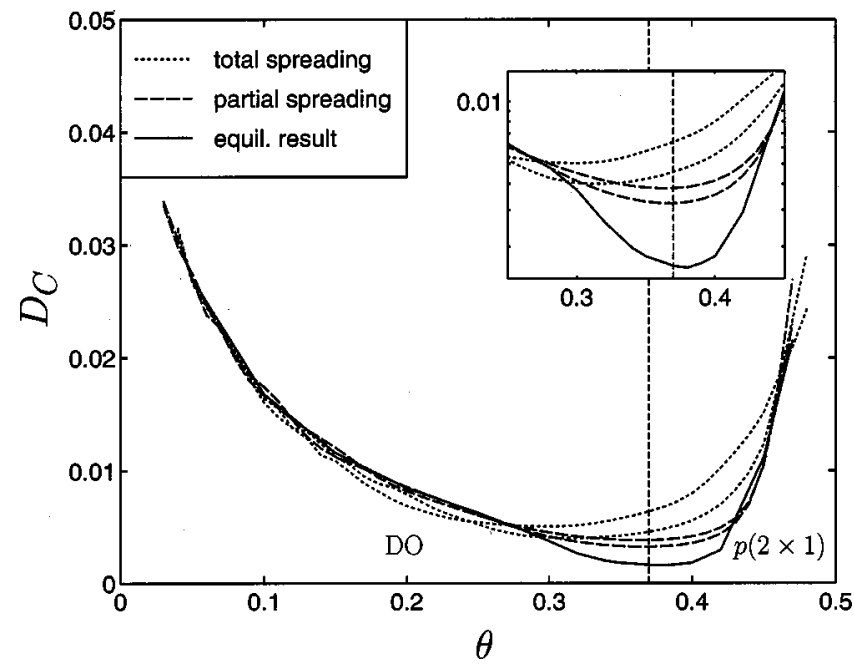

FIG. 5. Coverage dependence of the spatially averaged effective diffusion coefficient $D_{C}(\theta)$ as extracted from partial and total spreading studies at two different time scales at $T=590 \mathrm{~K}$. Upper and lower curves are for time scales of $t=50000$ and $250000 \mathrm{MC}$ steps, respectively. For the purpose of comparison, equilibrium data are also shown (Ref. 28). The regime around $\theta=0.37$ is shown in more detail in the inset (as a semilog representation).

equilibrium limit, with the deviations being largest close to the (equilibrium) phase boundary at $\theta \approx 0.37$ and within the ordered $p(2 \times 1)$ phase.

To compare directly with the full spreading data of Ref. 26, we show in Fig. 5 full and partial spreading data taken at two different time intervals (around 50000 and 250000 MCS). Details of the data around the phase transition boundary are furthermore shown in the inset of Fig. 5. Within the DO phase for $\theta \leqslant 0.15$, we find that there is essentially no difference between the different methods. For larger coverages the deviations between the two approaches increase and become strongest around $\theta \approx 0.37$. Comparison at this point reveals that the partial and full spreading results for $D_{C}(\theta)$ deviate by about $65 \%$ at $t=50000 \mathrm{MCS}$ and by about $43 \%$ at $t=250000 \mathrm{MCS}$. Further comparison to the equilibrium data reveals that in the case of partial spreading at $t$ $=250000 \mathrm{MCS}$, the deviation from true equilibrium behavior is about $95 \%$, which is considerably lower than the deviation by about $190 \%$ in the case of full spreading. Thus we can conclude that partial spreading data approach the equilibrium limit much more rapidly than the full spreading data.

Next, we consider the effect of the degree of ordering in the initial $\theta=\frac{1}{2}$ phase to the spreading profiles. To this end, let us first reconsider the results in Figs. 3(c) and 4(c). In the ordered phase $(0.37 \leqslant \theta \leqslant 0.50)$, we find that diffusion in the $x_{\|}$direction is considerably faster than in the direction of $x_{\perp}$ [see the snapshots in Figs. 2(a) and 2(b)]. Thus the quantitative behavior of $D_{C}(\theta)$ clearly depends on the initial state, although both initial phases are actually equally ordered.

In experimental systems the initial phase is typically a "spatially averaged", adsorbate system consisting of various ordered domains, or a disordered adsorbate layer after deposition of adatoms at a low temperature. For these cases, we consider two structures where the initial overlayer consists of two equal-size domains of the $p(2 \times 1)$ and $p(1 \times 2)$ phases, or where it is completely disordered (corresponding to an

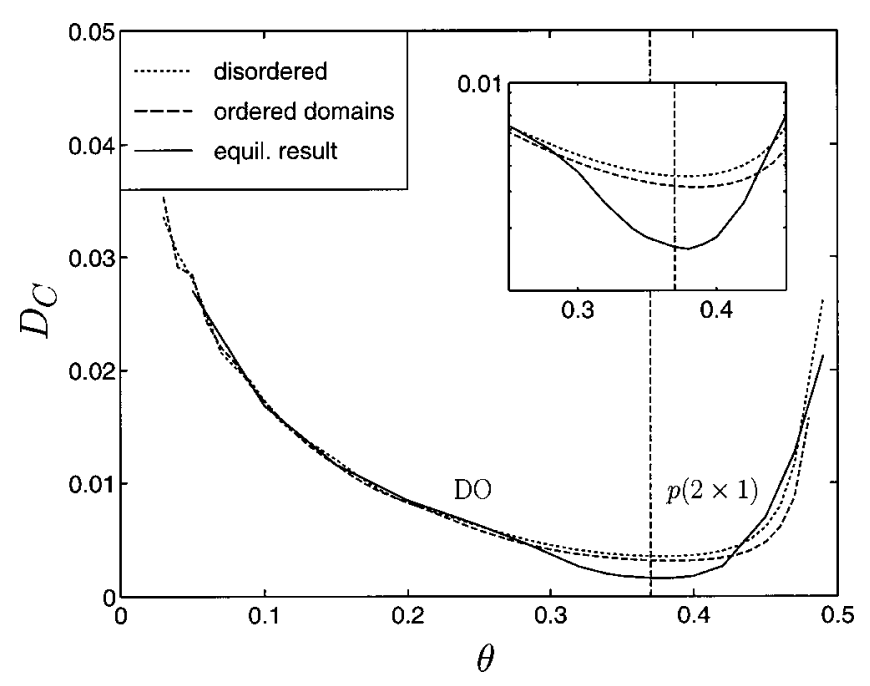

FIG. 6. Coverage dependence of the spatially averaged collective diffusion coefficient $D_{C}(\theta)$ as extracted from partial spreading using the two different initial states in Figs. 2(c) and 2(d). The temperature regime considered is $T=590 \mathrm{~K}$. Equilibrium data (Ref. 28) is shown with a solid line. The regime around $\theta=0.37$ is shown in more detail in the inset (as a semilog representation).

infinite temperature in the lattice-gas model). These situations are demonstrated in Figs. 2(c) and 2(d), respectively. The corresponding diffusion coefficients are shown in Fig. 6 around 50000 MCS. Perhaps surprisingly, we find that the behavior of the two submonolayer cases is very similar. Essentially there are no deviations in the disordered phase up to $\theta=0.25$, and even for larger coverages the differences are relatively minor.

The present findings allow us to suggest three major conclusions concerning submonolayer spreading experiments. First, if the initial state is ordered and has various degenerate ground states, then diffusion (spreading) rates along the possible directions may vary considerably. Second, regardless of the degree of ordering in the initial state, it takes only a relatively short time for the system to find a "near-equilibrium" state in which $D_{C}(\theta)$ behaves qualitatively as in equilibrium. Despite this, quantitative deviations from equilibrium behavior remain rather pronounced in ordered phases and close to phase boundaries. Reaching the true long-time behavior, in which the linear response theory is valid and Eq. (1) yields the true equilibrium result, therefore takes a very long time in environments where ordering plays a significant role. Nevertheless, the main conclusion is that deviations from equilibrium behavior are much weaker in submonolayer spreading when compared with full spreading studies, and therefore submonolayer experiments yield more reliable results.

\section{B. Time evolution of order parameter}

Previously, we have seen how nonequilibrium effects are manifested in the collective diffusion coefficient during the profile evolution process. To quantify the deviation from equilibrium during this process, we have additionally considered the time evolution of ordering within the $p(2 \times 1)$ phase in terms of the quantities 


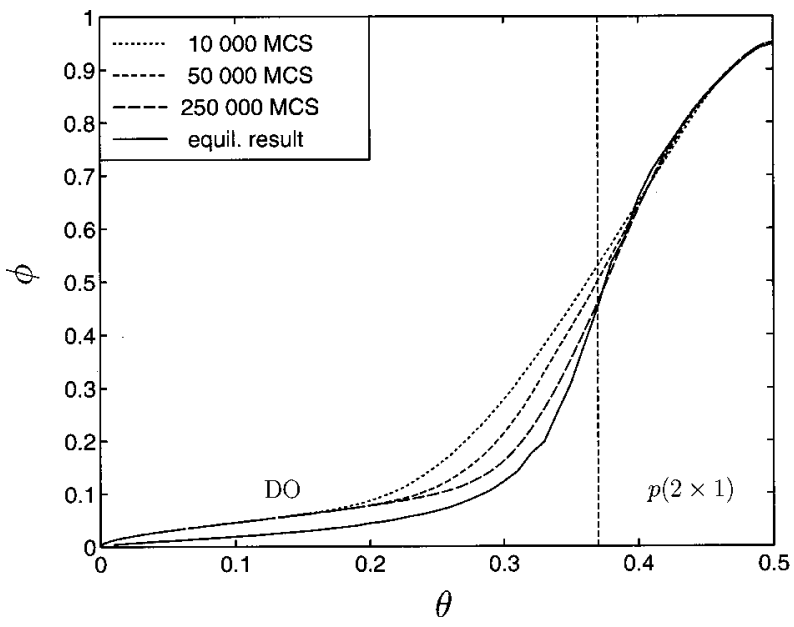

(a)

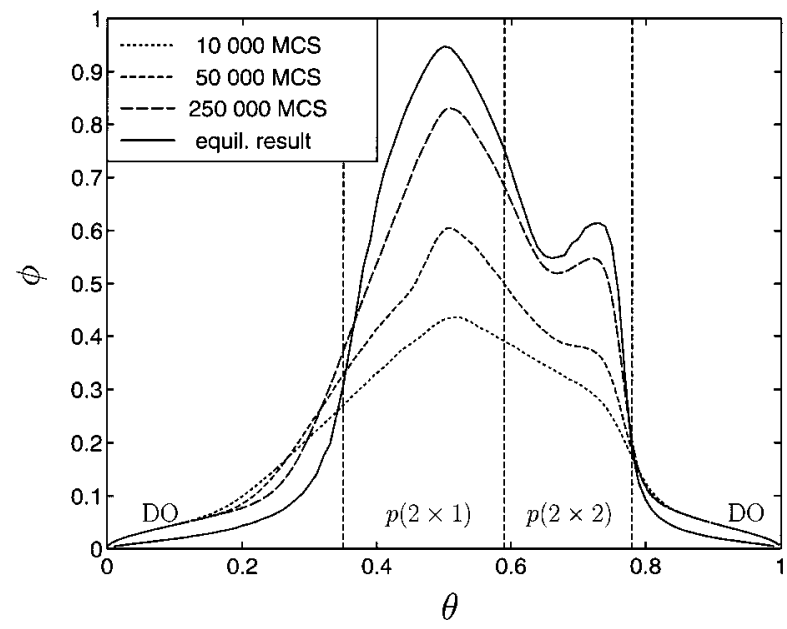

FIG. 7. Coverage dependence of the order parameter $\phi(t)$ at $T=590 \mathrm{~K}$ at three different time scales during profile spreading simulations. (a) Results for submonolayer spreading along the $x_{\|}$direction. (b) Results for spreading of a full monolayer. In both cases, equilibrium results have been given for comparison. Here, the spreading simulations have been made with $L_{y}$ $=200$, while equilibrium data corresponds to a system of size $60 \times 60$.

$$
\begin{aligned}
& \phi_{2 \times 1}(t) \equiv \frac{2}{L^{2}} \sum_{i, j=1}^{L} n_{i, j}(t)(-1)^{i}, \\
& \phi_{1 \times 2}(t) \equiv \frac{2}{L^{2}} \sum_{i, j=1}^{L} n_{i, j}(t)(-1)^{j},
\end{aligned}
$$

which define the order parameter components of the degenerate $p(2 \times 1)$ and $p(1 \times 2)$ phases in a square system of size $L \times L$, where $n_{i, j}(t)=0,1$ is the occupation variable of the lattice site at $(i, j)$ at time $t$. Since the two phases are energetically equivalent in the present system, we define the total order parameter as

$$
\phi(t) \equiv \sqrt{\phi_{2 \times 1}^{2}(t)+\phi_{1 \times 2}^{2}(t)}
$$

to quantify approach towards equilibrium. Equation (4) can also be used to determine the order parameter in equilibrium. In the profile spreading simulations, the time-dependent order parameter $\phi(t)$ has been averaged over successive strips of size $2 \times L_{y}$ to determine its coverage dependence.

Results shown in Fig. 7(a) are in full agreement with previous studies of $D_{C}(\theta)$. We find that in submonolayer spreading, the order parameter $\phi(t)$ approaches its equilibrium counterpart at long times, the deviation being largest close to $\theta \approx 0.3$. Therefore it is obvious that none of the coverage profiles in Figs. 3 and 4 are in the linear response regime of local equilibrium. The ordering process drives the system towards equilibrium and leads to the temporal dependence of $D_{C}(\theta)$ shown in Figs. 3(c) and 4(c).

In the case of full spreading, we again find that $\phi(t)$ approaches the equilibrium behavior at long times as shown in Fig. 7(b). In this case, deviations from equilibrium behavior are considerably stronger than in submonolayer spreading, being especially large within the ordered phases at intermediate coverages $0.20 \leqslant \theta \leqslant 0.80$. This is consistent with the fact that $D_{C}(\theta)$ in Fig. 5 for monolayer spreading deviates much more from the equilibrium diffusion data than $D_{C}(\theta)$ in the case of submonolayer spreading.

Let us now briefly discuss the temporal behavior of $\phi(t)$ in the context of ordering kinetics, a topic which has received plenty of attention in recent years. ${ }^{36}$ A typical example of an ordering experiment is a sudden temperature quench from a completely disordered adlayer to conditions that are characterized by an ordered equilibrium phase. Then, the system goes through an ordering process where the (average) characteristic domain size $\bar{R}(t)$ increases in time $t$ as $\bar{R}(t) \sim t^{x}$, and the idea is then to determine the exponent $x$ which depends on certain conservation laws. We have found above (see Fig. 7) that the order parameter increases in time, as expected for a system that tends towards equilibrium at long times. This is in agreement with theoretical predictions and experimental observations that $\bar{R}(t)$ (that is proportional to the order parameter) increases in time during a nonequilibrium ordering process. However, we feel that our results are not sufficient to make a quantitative prediction for $x$. This is due to time averaging in obtaining the order parameter profiles, which was necessary to get reasonable statistics from the spreading data.

Finally, we note that additional studies for the total energy of the system $E(\theta, t)$ are in full agreement with $\phi(t)$. We find that $E(\theta, t)$ approaches the asymptotic equilibrium behavior in the course of time, although deviations during the time scales considered are still rather significant.

\section{Phase boundaries}

We next discuss one aspect that is of general interest in studies of adsorption systems, namely the locations of phase boundaries between different phases. In the case of Boltzmann-Matano experiments, they can be determined from the minima of $D_{C}(\theta)$ provided that thermodynamic particle number fluctuations dominate the behavior of collective diffusion. To be more precise, the collective diffusion coefficient can be defined via the Green-Kubo equation ${ }^{37}$

$$
D_{C}(\theta)=\frac{1}{k_{B} T \theta \kappa_{T}} D_{\mathrm{cm}},
$$

where $D_{\mathrm{cm}}$ is the dynamic factor that describes the motion of the center-of-mass of the system. ${ }^{28,37}$ Our main interest in Eq. (5) concerns the compressibility $\kappa_{T}$, which is related to 
particle number fluctuations in a probe area, and which is expected to have a maximum at a (second-order) phase transition boundary. Thus, if the behavior of $D_{C}$ is dominated by particle number fluctuations, then the minima of $D_{C}$ provide information of phase boundaries. ${ }^{38}$ In previous studies, we have shown that this situation is indeed realized in the present model system at $T=590 \mathrm{~K} .^{28}$

Using this approach, results in Figs. 3(c) and 4(c) reveal that if one analyzes the Boltzmann-Matano data for full spreading at too early times, then the estimated locations of phase boundaries can deviate significantly from the actual equilibrium values. In the case of partial spreading, however, the effective phase boundaries are surprisingly close to their equilibrium limits. To confirm these conclusions, we also analyzed the time-dependent phase boundaries by considering the response of the order parameter, namely the quantity

$$
\Psi(t, T, \theta) \equiv\left|\left(\frac{\partial \phi(t, T, \theta)}{\partial \theta}\right)_{T}\right|,
$$

whose maxima characterize the locations of phase boundaries.

In the case of partial spreading, results in Fig. 8(a) show that the shape of $\Psi(t, T, \theta)$ as well as its maxima depend very slightly on the time scale under consideration. The nonequilibrium effects are not really significant, since the effective locations of phase boundaries are approximately correct even though they have been determined from nonequilibrium spreading profiles. The situation is very different in full spreading, where we also analyzed the coverage regime $0 \leqslant \theta \leqslant \frac{1}{2}$. In this case, results in Fig. 8(b) indicate that the maxima of $\Psi(t, T, \theta)$ depend strongly on the time regime considered. The maxima are now around $\theta \approx 0.30$ at $t$ $=10000 \mathrm{MCS}$, around $\theta \approx 0.32$ at $t=50000 \mathrm{MCS}$, and around $\theta \approx 0.36$ at $t=250000 \mathrm{MCS}$, thus approaching the equilibrium value $\theta \approx 0.37$ at long times.

We conclude that the Boltzmann-Matano approach can provide biased information of the locations of phase boundaries if one analyzes data at too early times. This is particularly true in full spreading, where the coverage profile goes through various ordered phases whose ordering processes are interfered by the ongoing mass flow. Only at long times, when the local density gradients become small and local equilibrium is established, the effective phase boundaries converge to their equilibrium limits. Evidently, since in submonolayer spreading there is only one (dis)ordering process that involves a smaller density gradient than in spreading of a full monolayer, the equilibration time is significantly shortened. Nevertheless, these observations, again, warrant particular care to be taken when profile spreading experiments are being carried out.

\section{SUMMARY AND DISCUSSION}

The profile evolution technique has been shown over the last 30 years to be an important experimental method for the study of surface diffusion. Together with the BoltzmannMatano analysis, it enables one to determine the coverage dependence of the collective diffusion coefficient $D_{C}(\theta)$ over the whole coverage range even from a single experi-

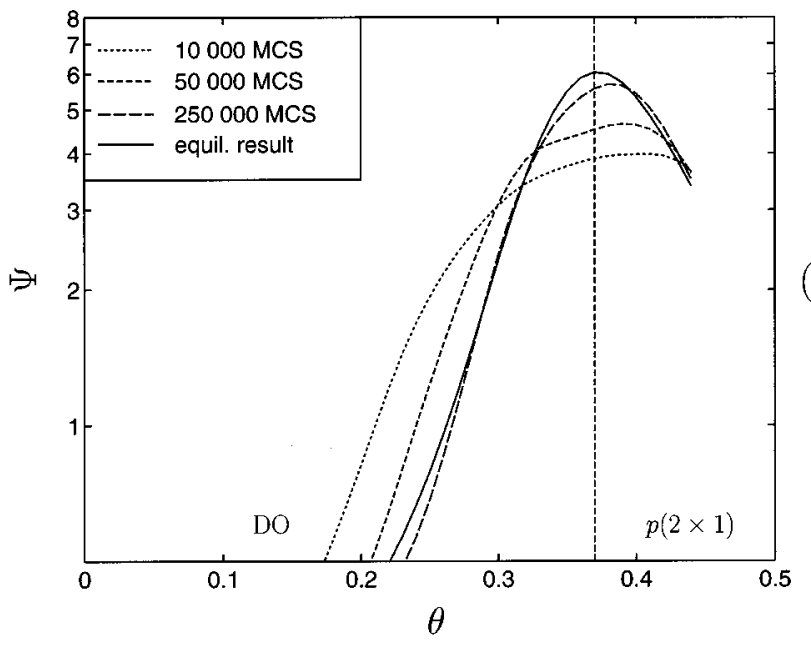

(a)

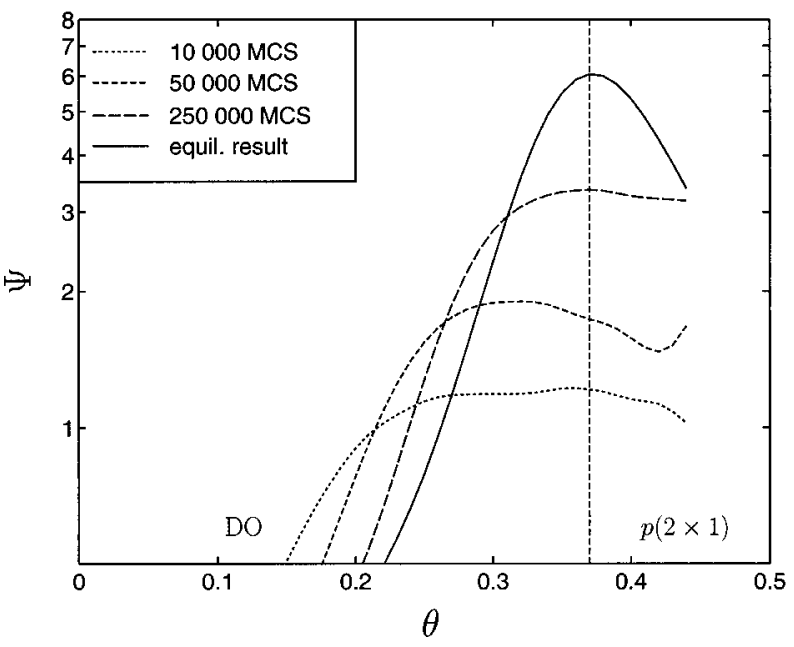

(b)

FIG. 8. Results for the response function $\Psi(t, T, \theta)$ at $0 \leqslant \theta \leqslant \frac{1}{2}$. The results correspond to order parameter profiles shown in Fig. 7, thus in (a) we consider submonolayer spreading, and in (b) we show results for the spreading of a full monolayer. In both cases, equilibrium results have been given for comparison.

ment. This property is a great advantage in experimental work and has attracted plenty of activities in recent years. However, the problem is that the Boltzmann-Matano analysis can be applied to any profile to obtain $D_{C}(\theta)$, and the equilibrium results are found only in the limit where scale invariance holds true, namely at long times when coverage profiles $\theta(x, t)$ collapse to a single scaling function when expressed as $\theta(x / \sqrt{t})$. Recent experimental measurements ${ }^{18,20}$ for $\theta(x / \sqrt{t})$ have indeed shown that the asymptotic long-time region is very difficult to achieve, and the observed deviations from scale invariance ${ }^{18,20}$ are a clear evidence of the nonequilibrium character of profile evolution. The obvious question that then arises is how closely related is the effective $D_{C}(\theta)$ to its equilibrium counterpart.

In the present work, we have addressed this question and considered the influence of nonequilibrium effects in the collective diffusion coefficient determined from coverage profiles during spreading. We have found that $D_{C}(\theta)$ is clearly time dependent and approaches its equilibrium limit only at very long times. The nonequilibrium effects are strongest within ordered phases and close to phase boundaries, while 
in disordered phases their role is essentially negligible. These effects are most easily faced in full spreading experiments, as the coverage profile starts from $\theta=1$ and goes through various ordered phases, while in submonolayer experiments the role of nonequilibrium is not as pronounced. This finding is well characterized by the locations of phase boundaries that strongly deviate from their equilibrium limits in the case of full spreading, and also by the diffusion barriers that are distinctly different from their equilibrium values. ${ }^{26}$

The present results highlight the important role of nonequilibrium effects played in profile evolution experiments, and in some other techniques such as laser-induced thermal desorption, which are based on creating a large density gradient and following the subsequent mass flow due to the diffusion process. The role of nonequilibrium effects is particularly important within ordered phases and close to phase boundaries, where deviations from equilibrium behavior are most pronounced. However, we note that nonequilibrium effects are just one of the problems associated with interpreting the diffusion data. Namely, profile evolution experiments are usually made over macroscopic distances, which implies that the effects due to impurities and surface defects cannot be entirely eliminated. Work to address the significance of these effects remains to be done.

\section{ACKNOWLEDGMENTS}

We wish to thank A. G. Naumovets, O. Trushin, and Z. Chvoj for useful discussions and comments. This work has, in part, been supported by the Academy of Finland through its Center of Excellence Program and by a grant from the European Union (I.V.).

${ }^{1}$ Surface Diffusion: Atomistic and Collective Processes, edited by M. C. Tringides (Plenum, New York, 1997).

${ }^{2}$ J. V. Barth, Surf. Sci. Rep. 40, 75 (2000).

${ }^{3}$ P. Jensen, Rev. Mod. Phys. 71, 1695 (1999).

${ }^{4}$ A. G. Naumovets and Yu. S. Vedula, Surf. Sci. Rep. 4, 365 (1985).

${ }^{5}$ M. C. Tringides, in The Chemical Physics of Solid Surfaces and Heterogeneous Catalysis: Phase Transitions and Adsorbate Restructuring of Metal Surfaces, edited by D. A. King and D. P. Woodruff (Elsevier, Amsterdam, 1994), Vol. 7, Chap. 6.

${ }^{6}$ R. Gomer, Rep. Prog. Phys. 53, 917 (1990)

${ }^{7}$ T. Ala-Nissila and S. C. Ying, Prog. Surf. Sci. 39, 227 (1992).

${ }^{8}$ A. Danani, R. Ferrando, E. Scalas, and M. Torri, Int. J. Mod. Phys. B 11, 2217 (1997).

${ }^{9}$ M. C. Tringides, P. K. Wu, and M. G. Lagally, Phys. Rev. Lett. 59, 315 (1987); P. K. Wu, M. C. Tringides, and M. G. Lagally, Phys. Rev. B 39, 7595 (1987).

${ }^{10}$ M. C. Tringides and R. Gomer, Surf. Sci. 265, 283 (1992).

${ }^{11}$ J. Haas, K. R. Roos, A. Jesina, and M. C. Tringides, Surf. Sci. 307-309, 465 (1994)
${ }^{12}$ I. Vattulainen, J. Merikoski, T. Ala-Nissila, and S. C. Ying, Surf. Sci. Lett. 366, L697 (1996).

${ }^{13}$ Z. Chvoj, J. Phys.: Condens. Matter 12, 2135 (2000).

${ }^{14}$ M. A. Załuska-Kotur, S. Krukowski, Z. Romanowski, and Ł. A. Turski, Surf. Sci. 457, 357 (2000).

${ }^{15}$ R. Butz and H. Wagner, Surf. Sci. 63, 448 (1977).

${ }^{16}$ E. Suliga and M. Henzler, J. Phys. C 16, 1543 (1983).

${ }^{17}$ A. G. Naumovets, M. V. Paliy, and Yu. S. Vedula, Phys. Rev. Lett. 71, 105 (1993).

${ }^{18}$ M. Šnábl, M. Ondřejček, V. Cháb, Z. Chvoj, W. Stenzel, H. Conrad, and A. M. Bradshaw, J. Chem. Phys. 108, 4212 (1998).

${ }^{19}$ A. T. Loburets, A. G. Naumovets, N. B. Senenko, and Yu. S. Vedula, Z. Phys. Chem. (Munich) 202, 75 (1997).

${ }^{20}$ A. T. Loburets, A. G. Naumovets, and Yu. S. Vedula, Surf. Sci. 399, 297 (1998).

${ }^{21}$ U. Albrecht, A. Otto, and P. Leiderer, Phys. Rev. Lett. 68, 3192 (1992); Surf. Sci. 283, 383 (1993).

${ }^{22}$ T. Ala-Nissila, S. Herminghaus, T. Hjelt, and P. Leiderer, Phys. Rev. Lett. 76, 4003 (1996); T. Hjelt, S. Herminghaus, T. Ala-Nissila, and S. C. Ying, Phys. Rev. E 57, 1864 (1998).

${ }^{23}$ C. Matano, Jpn. J. Phys. 8, 109 (1933).

${ }^{24}$ J. Crank, The Mathematics of Diffusion (Oxford University Press, Oxford, 1995).

${ }^{25}$ S. S. Mann, T. Seto, C. J. Barnes, and D. A. King, Surf. Sci. Lett. 261, 155 (1992).

${ }^{26}$ P. Nikunen, I. Vattulainen, and T. Ala-Nissila, Surf. Sci. Lett. 447, L162 (2000).

${ }^{27}$ I. Vattulainen, J. Merikoski, T. Ala-Nissila, and S. C. Ying, Phys. Rev. B 57, 1896 (1998)

${ }^{28}$ I. Vattulainen, S. C. Ying, T. Ala-Nissila, and J. Merikoski, Phys. Rev. B 59, 7697 (1999).

${ }^{29}$ G.-C. Wang, T.-M. Lu, and M. G. Lagally, J. Chem. Phys. 69, 479 (1978); C. R. Brundle and J. Q. Broughton, in The Chemical Physics of Solid Surfaces and Heterogeneous Catalysis: Chemisorption Systems, edited by D. A. King and D. P. Woodruff (Elsevier, Amsterdam, 1990), Vol. 3A, Chap. 3; K. E. Johnson, R. J. Wilson, and S. Chiang, Phys. Rev. Lett. 71, 1055 (1993).

${ }^{30}$ The precise location of the phase boundary depends on the system size, as expected. In the present case it is determined for a system of $60 \times 60$ lattice sites.

${ }^{31}$ I. Vattulainen, S. C. Ying, T. Ala-Nissila, and J. Merikoski, J. Chem. Phys. 111, 11232 (1999).

${ }^{32}$ I. Vattulainen, J. Merikoski, T. Ala-Nissila, and S. C. Ying, Phys. Rev. Lett. 80, 5456 (1998).

${ }^{33}$ I. Vattulainen, J. Merikoski, S. C. Ying, and T. Ala-Nissila, Europhys. Lett. 51, 361 (2000).

${ }^{34}$ H. C. Kang and W. H. Weinberg, J. Chem. Phys. 90, 2824 (1989).

${ }^{35}$ For every jump attempt, there are four possible directions of which two are independent. We have made equilibrium studies for single-particle transition rates in these two directions along the surface, thus obtaining $\Gamma_{x}$ and $\Gamma_{y}$. Then $\Gamma_{\perp}=\min \left\{\Gamma_{x}, \Gamma_{y}\right\}$ and $\Gamma_{\|}=\max \left\{\Gamma_{x}, \Gamma_{y}\right\}$.

${ }^{36}$ A. J. Bray, Adv. Phys. 43, 357 (1994).

${ }^{37}$ J. P. Boon and S. Yip, Molecular Hydrodynamics (Dover, New York, 1980).

${ }^{38}$ The compressibility can be determined by measuring the thermodynamic factor $\xi=1 /\left(k_{B} T \theta \kappa_{T}\right)$ using, for example, Gomer's fluctuation method (Ref. 6). 